\title{
Prognostic evaluation of COPD patients: GOLD 2011 versus BODE and the COPD comorbidity index COTE
}

\author{
Juan P de Torres, ${ }^{1}$ Ciro Casanova, ${ }^{2,3}$ Jose M Marín, ${ }^{4}$ Victor Pinto-Plata, ${ }^{5}$ \\ Miguel Divo, ${ }^{5}$ Javier J Zulueta, ${ }^{1}$ Juan Berto, ${ }^{1}$ Jorge Zagaceta, ${ }^{1}$ \\ Pablo Sanchez-Salcedo, ${ }^{1}$ Carlos Cabrera, ${ }^{5}$ Santiago Carrizo, ${ }^{4}$ Claudia Cote, ${ }^{6}$ \\ Bartolome R Celli ${ }^{7}$
}

For numbered affiliations see end of article.

\section{Correspondence to} Dr Juan P de Torres,

Pulmonary Department, Clínica Universidad de Navarra, Avda Pio XII, 36, Pamplona 31200, Spain; jpdetorres@unav.es

Received 20 May 2014 Accepted 1 June 2014 Published Online First 26 June 2014

\section{ABSTRACT}

Background The Global Obstructive Lung Disease (GOLD) 2011 revision recommends the multidimensional assessment of COPD including comorbidities and has developed a disease categories system (ABCD) attempting to implement this strategy. The added value provided by quantifying comorbidities and integrating them to multidimensional indices has not been explored. Objective Compare the prognostic value of the GOLD $A B C D$ categories versus the BMI, Obstruction, Dyspnea, Exercise (BODE) index, and explore the added prognostic value of comorbidities evaluation to this multidimensional assessment.

Methods From the patients who have been enrolled in the BODE study, we selected the most recent ones who had the available information needed to classify them by the ABCD GOLD categories. Cox proportional hazards ratios for all-cause mortality were performed for GOLD categories and BODE index. The added value of the comorbidity Copd cO-morbidity TEst (COTE) index was also explored using receiver operating curves (ROC) values.

Results 707 patients were followed for $50 \pm 30$ months including all degrees of airway limitation and BODE index severity. ABCD GOLD predicted global mortality (HR: $1.47 ; 95 \% \mathrm{Cl} 1.28$ to 1.70 ) as did the BODE index (HR: $2.02 ; 95 \% \mathrm{Cl} 1.76$ to 2.31). Area under the curve (AUC) of ROC for ABCD GOLD was 0.68; $(95 \% \mathrm{Cl} 0.64$ to 0.73$)$ while for the BODE index was $0.71(95 \% \mathrm{Cl}$ 0.67 to 0.76$)$. The $C$ statistics value was significantly higher for the observed difference. Adding the COTE index to the BODE index improved its AUC to 0.81 (95\% Cl 0.77 to 0.85$),\left(\chi^{2}=40.28, p<0.001\right)$.

Conclusions In this population of COPD patients, the BODE index had a better survival prediction than the $A B C D$ GOLD categories. Adding the COTE to the BODE index was complimentary and significantly improved outcome prediction.

\section{INTRODUCTION}

The 2011 revision of the Global initiative for chronic Obstructive Lung Disease (GOLD) strategy $^{1}$ recommends the assessment of patients with COPD using three different domains; severity of airflow obstruction, its impact on dyspnoea or patient's health status and risk of future events (exacerbations or hospital admissions). This new proposal implies an important conceptual step toward a multidimensional evaluation of COPD

\section{Key messages}

What is the key question?

- Which multidimensional index of COPD severity, the ABCD GOLD or the BODE Index is better to predict mortality over time, and what is the added value provided by quantifying selected comorbidities and integrating them to the multidimensional indices?

\section{What is the bottom line?}

- The BODE Index predicts mortality better than the GOLD ABCD grading and adding the COTE comorbidity Index to the BODE Index is complementary and provides an excellent predictive capacity for all-cause mortality in COPD patients.

\section{Why read on?}

- Multidimensional evaluation in COPD patients including comorbidities allow us to more precisely predict short and long-term all-cause mortality.

patients beyond the traditional assessment based on the degree of airway obstruction alone. ${ }^{2}$ Several tools have been proposed for the multidimensional evaluation of COPD patients. Among them, the BMI, Obstruction, Dyspnea, Exercise (BODE) index has been the most widely validated because it has shown a better predictive capacity for survival than the degree of airway obstruction measured by the $\mathrm{FEV}_{1} \%,{ }^{3}{ }^{4}$ it predicts hospitalisations, ${ }^{5}$ responds to interventions such as lung volume reduction surgery ${ }^{4}$ and pulmonary rehabilitation ${ }^{6}$ and relates well to health status. ${ }^{5}$

GOLD also acknowledges the frequent occurrence of comorbidities in these patients and their influence on hospitalisations and mortality. GOLD recommends that comorbidities should be actively looked for, and treated appropriately if present. However, it falls short of specifying which comorbidities and how to quantify them. ${ }^{1}$ Traditionally, the presence of comorbidities in COPD patients has been evaluated using a non-disease specific score such as the Charlson comorbidity score, developed for patients with cancer. ${ }^{7}$ More recently, Divo et $a l^{8}$ developed a 
COPD specific index the Copd cO-morbidity TEst or Copd cOmorbidity TEst (COTE) index that includes those comorbidities that impact on survival in COPD patients.

Although the scientific committee of the GOLD strategy acknowledges that the disease classification in $\mathrm{ABCD}$ categories is mainly for disease management and not for prognostic purposes, several studies have already compared its survivalpredictive capacity with the previous $\mathrm{FEV}_{1} \%$ based classifications. ${ }^{9-13}$ We planned the present study to compare the prognostic value of the revised GOLD categories with the BODE index and investigated the added prognostic value of the presence of comorbidities using the COTE index to the multidimensional assessment.

\section{METHODS}

The BODE cohort is an ongoing prospective, multicentre, observational study of subjects with COPD, recruited from pulmonary clinics in the USA and Spain. ${ }^{3}$ Between November 1997 and March 2009, a total of 1659 subjects from all five sites were enrolled in the study, and followed until either the time of death or to March 2012 (data of present analysis). The ethics committee at each of the participating centres approved the study, and all patients signed informed consent before enrolment.

The details of the BODE cohort inclusion and exclusion criteria have been previously described. ${ }^{3}$ In brief, COPD was defined on the basis of a history of smoking ( $>10$ pack-years) and on lung function test following the ATS/ERS standards. ${ }^{14}$ All patients were in clinically stable condition and receiving standard therapy. Subject were excluded if they had primary asthma, inability to take the lung function and 6 min walk tests, or any condition that could unacceptably increase the subject's risk of performing any of the testing.

Patients selected for the present study were those who also had information regarding the number of exacerbations occurred in the year previously to their enrolment. Exacerbations were defined by the added use of antibiotics, and/ or corticosteroids, or admission to the hospital related to worsening respiratory symptoms. We registered the number of subjects with $\geq 2$ exacerbations/years or $\geq 1$ hospitalisation/year. This allowed us to classify the patients into the ABCD categories proposed by the GOLD strategy. ${ }^{1}$ From the initial BODE cohort of 1659 patients, the most recently recruited $(n=707)$ had the information to classify them into the ABDC GOLD categories.

\section{Clinical and physiological parameters measurements}

The following information at the time of recruitment was obtained: age, gender, smoking status and history (pack-years). The Body Mass Index (BMI) was calculated as the weight in kilograms divided by height in square meters. Dyspnoea was evaluated using the mMRC scale. ${ }^{15}$ Pulmonary function tests were performed following ATS/ERS guidelines. ${ }^{14}$ The FEV1\%, BMI, 6MWD, and MMRC values were integrated into the BODE index that ranges in value from 0 to 10 . The Bode Index using that scale or divided in quartiles has been shown to predict mortality better than the FEV1 as described by Celli et al. ${ }^{3}$

\section{Comorbidities}

Comorbidities were systematically recorded through direct questioning for the following conditions: (1) those diseases included in the Charlson comorbidity index ${ }^{7}$ (19 comorbidities); (2) all comorbidities listed in the subject's medical record; or (3) those expressed during enrolment interview and subsequent visits. The diagnosis of a comorbidity was confirmed by either reviewing the patient's medication list, or when feasible by confirmatory tests available from their medical records. Conditions that had completely resolved were excluded (ie, pneumonia).

The COTE Index was constructed as previously described ${ }^{8}$ by scoring those same comorbidities that were associated with a statistically significant hazard of death. The sum of the points intends to capture the individual or combination of diseases affecting each patient. The scores range from 0 to 25 . A 1 point increase in the COTE Index is associated with an increased risk of death from COPD-related (HR, 1.13; 95\% CI 1.08 to 1.18; $\mathrm{p}<0.001)$ and non-COPD-related causes $(\mathrm{HR}, 1.18 ; 95 \% \mathrm{CI}$ 1.15 to $1.21 ; \mathrm{p}<0.001) .{ }^{8}$ COTE Index was also described according to the mortality risk in $<4$ points and $\geq 4$ points.

We followed the multidimensional evaluation recommended by the GOLD 2011 Revision. ${ }^{1}$ Airway limitation was evaluated by FEV $1 \%$ of predicted and categorised in $\geq 50 \%$ and $<50 \%$, symptoms by the mMRC scores, and categorised in mMRC $0-1$ and 2 or greater and exacerbation frequency in the previous year categorised in $0-1$ and 2 o greater. Unfortunately we do not have information on the COPD Assessment Test (CAT) or Clinical Copd Questionnaire scores or exacerbation with hospital admissions.

Patients were evaluated after enrolment and were seen every year or until death. The patient and family were contacted if the patient failed to return for appointments. Death from any cause was recorded and confirmed using death certificates in Spain and the social security registry in the USA.

\section{Statistical analysis}

Quantitative data with a normal distribution were expressed using the mean and the SD. Quantitative data with non normal distribution were described with the median and the IQR. Qualitative data were described using relative frequencies. The COTE and the BODE+COTE were handled as continuous scaled variable. Cox proportional hazards analysis was used to explore the association of ABCD GOLD categories and BODE quartiles with global mortality. Kaplan-Meier analysis was used to compare GOLD categories and BODE quartiles survival. The statistical significance was determined by the log-rank test. In order to determine how much more precise the BODE index and the BODE+COTE are than the ABCD categories to predict allcause mortality, we computed the $\mathrm{C}$ statistics for a model containing $\mathrm{ABCD}$ categories, $\mathrm{BODE}$ score or the BODE+COTE score as the sole independent variable. We compared the survival times and estimated the probabilities of death up to 24 and 50 months (mean value of follow-up). The null value for the $\mathrm{C}$ statistic is 0.5 , with a maximum of 1.0 (with higher values indicating being better predictive power). Significant levels for all tests were established as a two-tailed $\mathrm{p}$ value $\leq 0.05$. Calculations were made with SPSS V.20.0 (IBM, Chicago, Illinois, USA).

\section{RESULTS}

The baseline characteristics of the 707 patients are shown in table 1. Figure A1 of the Appendix shows the percentage of COPD patients with each BODE and COTE score. This COPD cohort includes patients of all degrees of airway limitation and disease severity evaluated by the BODE Index. One-third of them were still smoking with a low COTE Index and Charlson Score. All categories of the GOLD 2011 revision classification were represented with a higher percentage of categories $\mathrm{A}$ and D. Patients were followed for a median time of 50 months and 171 deaths occurred during that time.

Mortality during the follow-up time were for $\mathrm{ABCD}$ categories: A $11 \%$, B 16\%, C 23\% and D 42\%; for BODE quartiles: 
Table 1 Baseline characteristics of COPD patients included in the study

\begin{tabular}{ll}
\hline Clinical and physiological characteristics & $\mathrm{N}=707$ \\
\hline Age in years: mean (SD) & $64(9)$ \\
Gender (male \%) & 80 \\
BMI in kg/m²: mean (SD) & $27(5)$ \\
Pack-years: median (IQR) & $50(40-74)$ \\
Current smoking (\%) & 33 \\
Charlson Index: mean (SD) & $1.8(1.2)$ \\
Range & $1-15$ \\
COTE Index: mean (SD) & $0.6(1.5)$ \\
Range & $0-10$ \\
COTE <4 (\%); COTE $\geq 4(\%)$ & $75 ; 25$ \\
FEV ${ }_{1} \%:$ mean (SD) & $55(21)$ \\
FVC\%: mean (SD) & $88(22)$ \\
FEV 1 /FVC\%: mean (SD) & $51(14)$ \\
No exacerbations: mean (SD) & $1(1.4)$ \\
2007 GOLD: I, II, II, IV (\%) & $14,40,33,13$ \\
2011 GOLD: A, B, C, D (\%) & $36,10,23,31$ \\
BODE quartiles: Q1, Q2, Q3, Q4 (\%) & $58,22,11,9$ \\
Follow-up time in months: median (IQR) & $50(23-85)$ \\
\hline
\end{tabular}

BMI, body mass index; BODE, BMI, Obstruction, Dyspnea, Exercise; COTE, Copd cOmorbidity TEst (COTE); GOLD, Global Obstructive Lung Disease.

Q1 14\%, Q2 30\%, Q3 41\% and Q4 62\% and for COTE Index: $<40.7 \%$ and $\geq 422 \%$.

There was no association between the BODE and COTE Indices (rho Spearman coefficient $=-0.05, p=0.15$ ). The ABCD GOLD is strongly associated with BODE (rho Spearman coefficient $=-0.83, \mathrm{p}<0.001)$.

Table 2 shows the Cox proportional HRs for ABCD GOLD, BODE quartiles, COTE Index and Charlson Score. All tools showed a significant but variable association with the risk of mortality in this selected population.

Figure 1 shows the Kaplan-Meier survival curves for ABCD GOLD categories in panel A and for BODE quartiles in panel B. Although statistical significance was again found in both comparisons, the BODE showed a better separation of the curves with higher quartiles associating with worse survival. Interestingly, those patients in category C of GOLD had a better survival than those in grade $\mathrm{B}$.

Figure 2 shows the receiver operating curves (ROC) curves for each of the parameters and their respective area under the curve (AUC). The BODE Index has the best predictive capacity of all of the single explored parameters with an AUC of 0.71 (95\% CI 0.67 to $0.76, p<0.001$ ). Combining both assessments (multidimensional+comorbidity evaluation) using the BODE Index +COTE Index had the best predictive power for global mortality in this population.

Table 2 Cox proportional hazards analysis for each multidimensional tool and comorbidity index o score

\begin{tabular}{llllll}
\hline Parameters & B & p Value & $\boldsymbol{\beta}$ & $95 \% \mathrm{Cl}$ & \\
\hline GOLD ABCD & 0.38 & $<0.001$ & 1.47 & 1.28 & 1.70 \\
BODE quartiles & 0.70 & $<0.001$ & 2.02 & 1.76 & 2.31 \\
COTE & 0.22 & $<0.001$ & 1.25 & 1.18 & 1.32 \\
Charlson & 0.13 & $<0.001$ & 1.13 & 1.07 & 1.20
\end{tabular}

BODE, BMI, Obstruction, Dyspnea, Exercise; COTE, Copd cO-morbidity TEst (COTE); GOLD, Global Obstructive Lung Disease.
Figure 3 shows the ROC curves for ABCD GOLD, COTE Index and BODE+COTE and their respective AUC at 24 months of follow-up. The statistical comparison of the AUC for ABCD GOLD versus BODE+COTE was statistically significant $\left(\chi^{2}=40.28, \mathrm{p}<0.001\right)$. At this earlier time point of 24 months, the BODE was still superior to the ABCD GOLD categories, and if added to the COTE Index, preserved its strong predictive capacity.

\section{DISCUSSION}

This study shows that the BODE Index is a better tool to evaluate the severity of COPD and ultimate risk of death at short and long term than the proposed multidimensional ABCD GOLD category classification. As the recent GOLD 2011 revision recommends, ${ }^{1}$ we also show that a combined multidimensional assessment including the evaluation of comorbidities using the COPD-specific COTE Index improves the ability to prognosticate survival in these patients.

Although the ABCD GOLD categories are meant to guide patient management and not their prognosis, it would be desirable that a novel category system were superior to the one to be supplanted and had the capacity to predict outcome.

Based on this assumption several studies have already explored the prognostic qualities of the ABCD system in different populations. ${ }^{9-13}$ The Spanish multicenter COCOMICS study ${ }^{9}$ included 3,633 patients with COPD with all degrees of severity, recruited from different levels of care (primary care and specialists) accumulating a total of 15878 persons-year. In that cohort, the ABCD categories did not improve the prognostic capacity of the $2007 \mathrm{FEV}_{1}$ based GOLD classification. ${ }^{2}$ Similarly, results from a cohort of 912 patients followed in Norway over 9 years ${ }^{12}$ observed no difference in the capacity to predict mortality or exacerbations leading to hospitalisations between the new ABCD and the old GOLD classification. ${ }^{2}$ More recently, the results of the HUNT cohort have also been the subject of a study. ${ }^{13}$ This cohort consists of 1540 people with postbronchodilator COPD who participated in the Norwegian Nord-Trøndelag Health Study between 1995 and 1997, and were followed-up on all-cause mortality until May 2012. This study population had more patients with milder COPD (61\% in group A, 18\% in group B, 12\% in group C and $10 \%$ in group D) than the previous studies. By contrast with spirometric GOLD grades where mortality increased with higher grade of severity, there were only minor differences in mortality between categories $\mathrm{A}$ and $\mathrm{B}$, and between categories $\mathrm{C}$ and $\mathrm{D}$. The authors concluded that the ABCD categories were substantially inferior to the old lung function-based classification to predict mortality. Agusti et al ${ }^{11}$ explored the ECLIPSE cohort of COPD patients with all degrees of severity followed for 3 years, and confirmed that those classified in grade $\mathrm{B}$ had a poorer survival than those classified in group C. However, no comparison was made with the old 2007 GOLD spirometric classification. Finally, the Copenhagen City Heart Study did compare the predictive capacity of the old and new GOLD classification $^{10}$ in a large population most of whom had mild to moderate degree of airway limitation. In that study, the ABCD categories did stratify patients with differences in survival, but once again demonstrated that those classified as category B had a worse prognosis than those in category C. Importantly, there was no advantage of the new over the old classification in the ability to predict mortality.

Although comparisons of the prognostic capacity of the GOLD 2011 revision classification have been made against the old classification based solely on $\mathrm{FEV}_{1}$, the present study is 
Figure 1 Kaplan-Meier survival curves for (A) $A B C D$ Global Obstructive Lung Disease (GOLD) categories and for (B) BMI, Obstruction, Dyspnea, Exercise (BODE) quartiles.
A

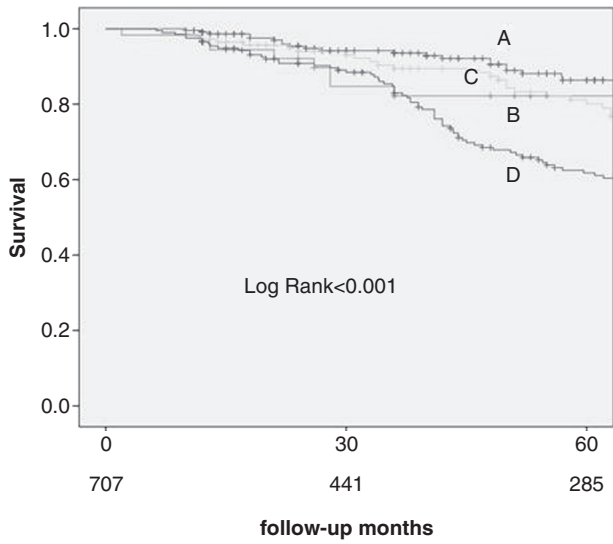

ABCD $G O L D$
B

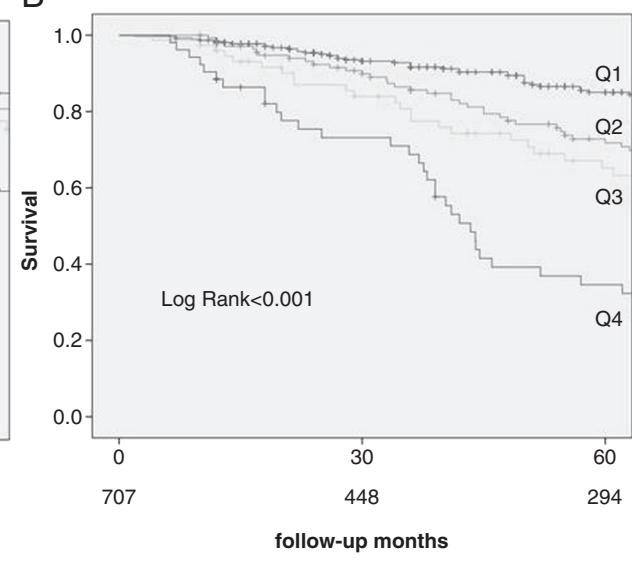

BODE Quartiles the first one comparing the ABCD system with another multidimensional assessment tool, the BODE Index. The results show that evaluation performed by the BODE Index is superior to that of the ABCD GOLD categories at short (24 months) and long-term (50 months) evaluation (figure 1). It has already been shown that the BODE Index predicts mortality better than the $\mathrm{FEV}_{1} \%{ }^{3}{ }^{4}$ The current study also confirms that the evaluation of the extra domains of BMI and the $6 \mathrm{~min}$ walk distance improves the predictive capacity of symptoms (in this case only evaluated by MRC) and exacerbation history in the previous year.

A detailed analysis of the ABCD GOLD prognostic power at 50 months (the mean time of follow-up for this study) in figure $1 \mathrm{~A}$, shows two groups of patients with different survival: $A$ and $C$ versus $B$ and $D$. In fact, integrating the patients in grades $\mathrm{A}+\mathrm{C}$ versus $\mathrm{B}+\mathrm{D}$ the Cox proportional $\mathrm{HR}$ for mortality is $2.0595 \%$ CI 1.46 to $2.87, \mathrm{p}<0.001$ for the latter group. This implies that the more symptomatic patients (higher mMRC in this study), are the ones with worse survival, especially if they also have frequent exacerbations $(>2)$ in the previous year (grade B). These results are in agreement with the observations made by Lange et $a l^{10}$ that showed that the presence of symptoms (evaluated with the mMRC scale) was an important driver of mortality in their analyses.

Although differences in population type and degree of severity with the other comparative studies could explain some differences found in survival of COPD patient according to the different classification systems, none of the studies were completed up to date, which include cohorts with large number of patients in all cells of the ABCD categories system, have shown an advantage of the proposed system over the old $\mathrm{FEV}_{1}$ based system. Our findings are in agreement with these previous studies. An important strength of the present study is the high proportion of patients in category $\mathrm{C}$, which allowed us to properly define their comparative survival with the already proven good prognosis of category A and bad prognosis of category

\section{ABCD GOLD}

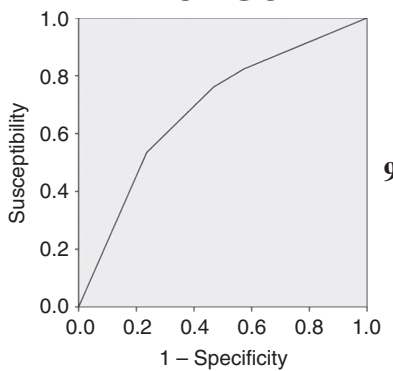

0.68 95\% CI: 0.64 to 0.73

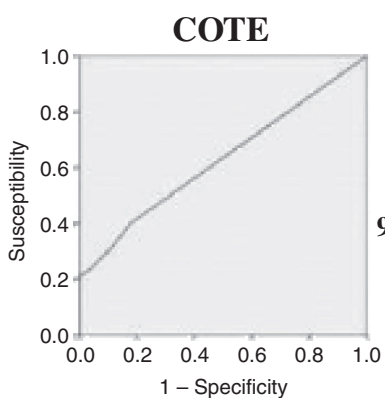

BODE

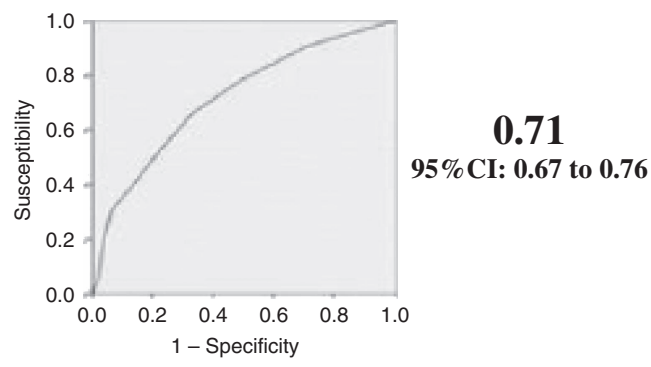

BODE + COTE

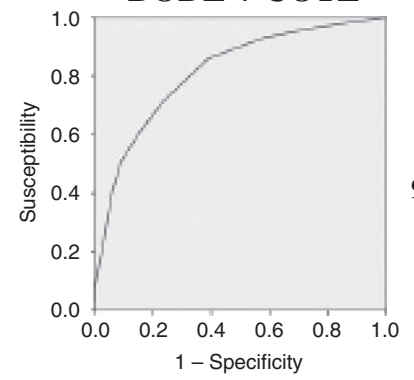

Figure 2 Receiver operating curves (ROC) in the BODE cohort for the ABCD category system, the BMI, Obstruction, Dyspnea, Exercise (BODE) index, the Copd CO-morbidity TEst (COTE) comorbidity Index, and the combination of the BODE+COTE Index and their respective area under the curve (AUC). 
ABCD GOLD

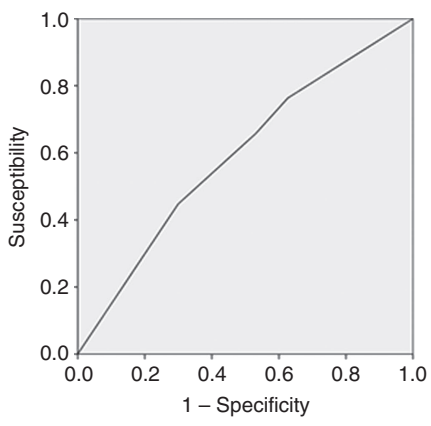

AUC $=0.59$

95\% CI: 0.50 to 0.68

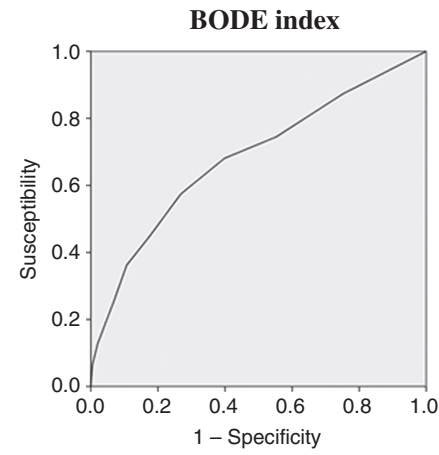

$\mathrm{AUC}=\mathbf{0 . 6 8}$

95\% CI: 0.59 to 0.77

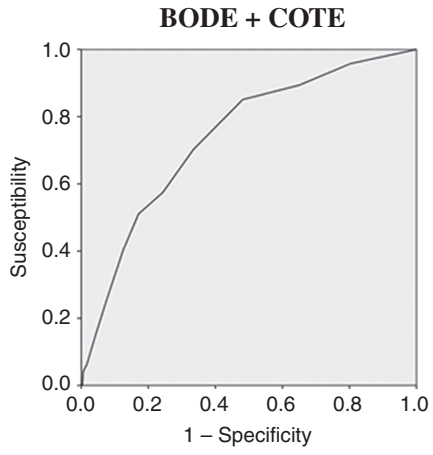

$\mathrm{AUC}=\mathbf{0 . 7 4}$

95\% CI: 0.67 to 0.81

Figure 3 Mortality receiver operating curves (ROC) curves in the BMI, Obstruction, Dyspnea, Exercise (BODE) cohort for ABCD Global Obstructive Lung Disease (GOLD), BODE Index and BODE+Copd CO-morbidity TEst (COTE) and their respective area under the curve (AUC) at 24 months of follow-up.

D. Additionally, we confirmed the previous observation that patients in category B seem to have a long-term worse prognosis than those in category $\mathrm{C}$.

A very important concept introduced by the recently GOLD 2011 revision was the need to actively look for associated comorbidities so they can be properly treated. However GOLD does not specify the nature of the comorbidities to be studied or provide a measure of quantification of comorbidities that impact on COPD survival. Traditionally, the Charlson score (initially developed to evaluate comorbidites in oncologic patients) ${ }^{7}$ has been used to quantify the added risk for mortality provided by a select group of 19 comorbidities. Recently, Divo $e t a l^{8}$ developed a simple multimorbidity index using a set of 12 comorbidities documented in 1664 COPD patients with all degrees of severity and integrated them into the composite COTE index. We extended those findings by adding the COTE Index to the BODE Index, a finding previously reported in the study by Divo et al. ${ }^{8}$ The present study results also show that this combination provided an excellent predictive capacity for risk of death over the 50 months of the study $(\mathrm{AUC}=0.81)$ far superior to the values provided by each single index alone. This finding provides evidence that the simple quantification of a selected group of comorbidites using the COTE Index has important prognostic value at short and long term. The COTE Index is simple to implement and could help clinicians and researchers quantify the severity of the comorbid conditions.

The present study has several limitations. First, these findings could only be translated to the type of patients here presented, mostly patients attending pulmonary clinics. Other studies with different type of patients and from different settings should confirm the external validity of our findings. Second, symptoms evaluation in the present study was performed with the degree of dyspnoea measured by the modified MRC Scale. As already discussed by Agusti et al, ${ }^{16}$ several studies have confirmed that the use of other tools like the proposed $\mathrm{CAT}^{17}$ or the Clinical COPD Questionnaire $(\mathrm{CCQ})^{18}$ could change patient grade assignment with their potential impact on survival. ${ }^{19}$ However, the mMRC Scale is a solid independent prognostic tool for COPD survival. ${ }^{20}$ Third, the cohort consisted primarily of men and, therefore, its conclusions have to be validated in women. However, we have already shown the validity of the BODE index in women with COPD. ${ }^{21}$ Finally, we used all-cause mortality as the outcome because it is the more solid outcome of the disease and could be used to compare our results with those of other studies. We acknowledge that it could have been interesting to know the exact cause of death of each ABCD GOLD category, but this was not the aim of the study.

In summary, in this population of COPD patients attending pulmonary clinics, the BODE Index predicted survival better than the newly proposed GOLD ABCD categories, at short and long-term follow-up periods. Adding the COTE comorbidity Index to the BODE Index is complementary and provides an excellent predictive capacity for all-cause mortality. These findings should be reproduced in other populations.

\section{Author affiliations}

${ }^{1}$ Pulmonary Department, Clínica Universidad de Navarra, Pamplona, Spain ${ }^{2}$ Pulmonary Department, Hospital Ntra Sra de Candelaria, Tenerife, Spain ${ }^{3}$ Respiratory Research Unit, Hospital Ntra Sra de Candelaria, Tenerife, Spain ${ }^{4}$ Pulmonary Department, Hospital Universitario Miguel Servet, Zaragoza, Spain

${ }^{5}$ Pulmonary Department, Hospital Universitario Dr Negrin, Las Palmas, Spain

${ }^{6}$ Pulmonary Department, Bay Pines VA Medical Center, St Petersburg, Florida, USA

${ }^{7}$ Pulmonary Department, Brigham and Women's Hospital. Harvard Medical School Boston, Boston Massachusetts, USA

Contributors Conception and design: JPdT, CC, JMM, CC, VP-P, MD and BRC. Analysis and interpretation: JPdT, CC, JMM, CC, VP-P, BRC, PS-S, JZ, CC and MD. Drafting the manuscript for important intellectual content: JPdT, CC and BRC. JPdT is the guarantor of the paper, taking responsibility for the integrity of the work as a whole, from inception to published article.

Competing interests None.

Ethics approval 043/2010 Certificado Comite de Etica CUN.

Provenance and peer review Not commissioned; internally peer reviewed.

\section{REFERENCES}

1 Vestbo J, Hurd SS, Agustí AG, et al. Global strategy for the diagnosis, management, and prevention of chronic obstructive pulmonary disease: GOLD executive summary. Am J Respir Crit Care Med 2013;187:347-65.

2 Rabe KF, Hurd S, Anzueto A, et al.; Global Initiative for Chronic Obstructive Lung Disease. Global strategy for the diagnosis, management, and prevention of chronic obstructive pulmonary disease: GOLD executive summary. Am J Respir Crit Care Med 2007; 176:532-55.

3 Celli BR, Cote C, Marin JM, et al. The Body Mass Index, Airflow Obstruction, Dyspnea, Exercise Performance (BODE) index in chronic obstructive pulmonary disease. N Engl J Med 2004;350:1005-12.

4 Martinez FJ, Foster G, Curtis JL, et al.; NETT Research Group. Predictors of mortality in patients with emphysema and severe airflow obstruction. Am J Respir Crit Care Med 2006;173:1326-34

5 Marin JM, Cote CG, Diaz O, et al. Prognostic assessment in COPD: health related quality of life and the BODE index. Respir Med 2011;105:916-21.

6 Cote CG, Celli BR. Pulmonary rehabilitation and the BODE index in COPD. Eur Respir J 2005;26:630-6. 
7 Charlson ME, Pompei $\mathrm{P}$, Ales $\mathrm{KL}$, et al. A new method of classifying prognostic comorbidity in longitudinal studies: development and validation. J Chronic Dis 1987;40:373-83.

8 Divo M, Cote C, de Torres JP, et al.; BODE Collaborative Group. Comorbidities and risk of mortality in patients with chronic obstructive pulmonary disease. Am J Respir Crit Care Med 2012;186:155-61.

9 Soriano JB, Alfageme I, Almagro $\mathrm{P}$, et al. Distribution and prognostic validity of the new Global Initiative for Chronic Obstructive Lung Disease grading classification. Chest 2013;143:694-702.

10 Lange $\mathrm{P}$, Marott JL, Vestbo J, et al. Prediction of the clinical course of chronic obstructive pulmonary disease, using the new GOLD classification: a study of the general population. Am J Respir Crit Care Med 2012;186:975-81.

11 Agusti A, Edwards LD, Celli B, et al.; ECLIPSE Investigators. Characteristics, stability and outcomes of the 2011 GOLD COPD groups in the ECLIPSE cohort. Eur Respir J 2013;42:636-46

12 Johannessen A, Nilsen RM, Storebø M, et al. Comparison of 2011 and 2007 Global Initiative for Chronic Obstructive Lung Disease Guidelines for Predicting Mortality and Hospitalization. Am J Respir Crit Care Med 2013;188:51-9.

13 Leivseth L, Brumpton BM, Lund Nilsen TI, et al. GOLD classifications and mortality in chronic obstructive pulmonary disease: the HUNT Study, Norway. Thorax 2013:68:914-21.
14 Celli BR, MacNee W. Standards for the diagnosis and treatment of patients with COPD: a summary of the ATS/ERS position paper. Eur Respir J 2004;23:932-46.

15 Mahler D, Weels C. Evaluation of clinical methods for rating dyspnea. Chest 1988:93:580-6.

16 Agusti $A$, Hurd S, Jones $P$, et al. FAQs about the GOLD 2011 assessment proposal of COPD: a comparative analysis of four different cohorts. Eur Respir J 2013;42:1391-401.

17 Jones PW, Harding G, Berry P, et al. Development and first validation of the COPD Assessment Test. Eur Respir J 2009:34:648-54.

18 van der Molen T, Willemse BW, Schokker S, et al. Development, validity and responsiveness of the Clinical COPD Questionnaire. Health Qual Life Outcomes 2003;1:13.

19 Casanova C, Marin JM, Martinez-Gonzalez C, et al.; COPD History Assessment In Spain (CHAIN) Cohort. New GOLD classification: longitudinal data on group assignment. Respir Res 2014;15:3.

20 Nishimura K, Izumi T, Tsukino M, et al. Dyspnea is a better predictor of 5-year survival than airway obstruction in patients with COPD. Chest 2002;121: $1434-40$.

21 de Torres JP, Cote CG, López MV, et al. Sex differences in mortality in patients with COPD. Eur Respir J 2009:33:528-35.

\section{APPENDIX}
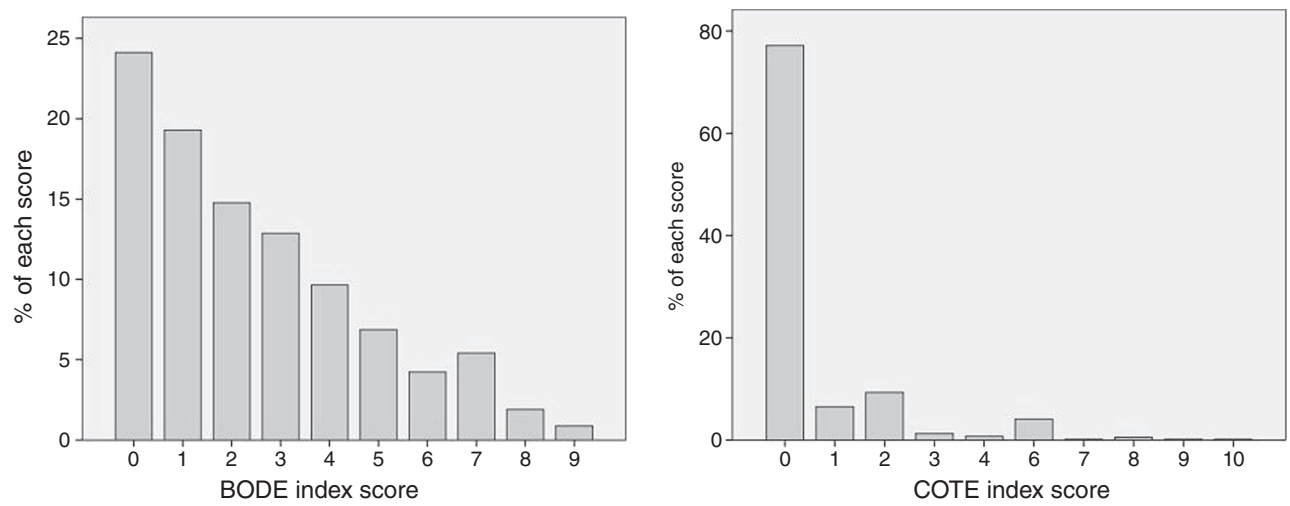

Figure A1 Percentage of COPD patients with each BMI, Obstruction, Dyspnea, Exercise (BODE) and Copd cO-morbidity TEst (COTE) score. 